\title{
Assessment and management of refractory breathlessness in interstitial lung disease
}

\author{
Lucy Speakman, Respiratory Nurse, Oxford Health NHS Foundation Trust \\ Helen Walthall, Oxford Brookes University
}

\begin{abstract}
Interstitial lung disease (ILD) refers to a cluster of fibroinflammatory conditions. There are limited treatment options and most patients have severe dyspnoea. The prognosis is poor. This study aims to evaluate current literature on the assessment and management of refractory breathlessness in ILD. Few tools are available to assess dyspnoea in advanced respiratory disease. Holistic assessment requires a combination of tools but there are few disease specific tools. The role of opioids is well established in the reduction of breathlessness, but there is insufficient evidence that benzodiazepines are beneficial.

Non-pharmcolological breathlessness intervention services can give patients mastery of their disease, reduced distress due to breathlessness and were more cost effective. More research on holistic interventions for use in advanced disease needs to be done. Patient-reported outcome measures could elicit valuable evidence to describe the benefit of breathlessness management services in advanced respiratory disease.
\end{abstract}

\section{KEY POINTS}

- interstitial lung disease is a devastating disease with breathlessness impacting on quality of life.

- Assessment tools can enable meaningful measurement of breathlessness.

- Nurses are well placed to support patients with non-pharmacological interventions.

- Mastery as an outcome measure can capture beneficial interventions for patients.

- A multidisciplinary approach to patient care can improve outcomes.

\section{KEY WORDS}

assessment tools, refractory breathlessness, holistic management, end of life care, mastery 
Interstitial lung disease (ILD) is a term that refers to over 200 different diseases. There are approximately 5000 new cases of ILD diagnosed each year in the UK (British Thoracic Society, 2015/16), the incidence of ILD is greater in men and has a mean survival rate of 3 years postdiagnosis, which is a worse prognosis than many cancers (e.g. prostate or bladder cancer). Interstitial means the disease affects the interstitium: the lace-like network of tissue that supports the air sacs in the lungs. Pulmonary fibrosis is a type of ILD. The disease results a combination of scarring and inflammation in the lungs, which causes the lungs to become stiffer and lose their elasticity; they are less able to inflate, which reduces the efficiency of breathing (British Lung Foundation, 2017) There has been limited research undertaken into the assessment and symptom management of this group of patients. Although advancing drug development enables disease progression to be slowed (National Institute for Health and Care Excellence (NICE), 2013; 2016), the symptom burden of breathlessness is universal at the end-stage of the disease. Chronic refractory breathlessness refers to breathlessness that is persistent and continues despite optimising treatment for the underlying condition (Currow et al, 2013), and is a complex symptom that has been linked with emotion and anxiety (Johnson et al, 2014). Community-based respiratory nurses, within the multidisciplinary team, are in an ideal position to support such patients and their informal caregivers through a range of pharmacological and non-pharmacological interventions, which could help improve their quality of life. This paper will discuss such issues and use a case from practice to illustrate the challenges for community nurses. The clinical case John (pseudonym assigned as per the Nursing and Midwifery Council (NMC) Code confidentiality clause) is a 74 year old gentleman diagnosed with atypical idiopathic pulmonary fibrosis, which is severe and progressive. He was first diagnosed in 2014, having not previously been breathless, and now (2017) suffers from refractory breathlessness. John's breathlessness was assessed using the Borg Scale (Borg, 1982), and scored 7/10 at rest and $10 / 10$ on exertion. On physical examination, John used pursed lip breathing at rest and his scalene, sternomastoid and trapezium accessory muscles to support his inspiration, he was only able to speak in broken sentences. Psychologically, John was finding it hard to 'cope' with his symptoms and diagnosis and gained support from the wider multidisciplinary team at the local hospice every week. When discussing John's symptoms he admitted to feeling very frightened and anxious at the degree of breathlessness he endured on a daily basis. The hospice team felt John was slowly accepting his short prognosis, but that he remained reluctant to talk about end of life and therefore advanced care planning discussions have been limited. From managing John's care, a number of issues arose: firstly, refractory breathless assessment and management; secondly, psychological support required for John; and thirdly, any treatment options to improve his quality of life.

\section{Refractory breathlessness as a symptom}

Understanding the physiological components of breathlessness can help to identify effective strategies for intervention. Research has historically focused on optimising lung function to reduce breathlessness but there is increasing interest in exploring the brain mechanisms underlying dyspnoea perception (Herigstad et al, 2011).

Parallels between the breathlessness pathway and the chronic pain pathway have been drawn, both being driven by both cognitive and affective factors ( Herigstad et al, 2011). Pain, like dyspnoea, is a highly subjective sensation often associated with negative emotions (Gracely et al, 2007). It is proposed that repeated exposure to breathlessness worsens our perception of it, suggesting a clear relationship of physical and emotional responses to breathlessness (Jansens et al, 2009). 


\section{Assessment of refractory breathlessness in interstitial lung disease}

The primary tool used for gathering data in John's study was through narrative assessment, which enabled clear identification of problems and needs specific to John. This was performed through open questioning and was informed by Roper, Logan and Tierney's (1983) activities of daily living (ADL). Further objective assessment was undertaken using a modified Borg Visual Analogue Scale (VAS), which is the current respiratory assessment tool used in practice to assess John's level of breathlessness. Borg is a generic assessment tool, with the modified Borg Scale being a valid and reliable VAS tool to measure breathlessness. However, the use of Borg is not without criticism. Wilson and Jones (1989) argued as it is not a disease-specific tool, and is limited to measuring intensity, its strength as an effective assessment tool of refractory breathlessness is questionable. This is further supported by McCord and Chronin- Stubbs (1992) who identified that the Borg tool did not offer a framework for wider holistic assessment, planning or evaluation of care, which are essential components for the assessment of refractory breathlessness in this specific group of patients.

The modified Borg is a unidimensional assessment tool that was originally developed by Borg (1982) to assess the impact of perceived exertion on breathlessness. The tool is used extensively within clinical practice to measure the intensity of exertion on the sensation of breathlessness. This has limited relevance within refractory breathlessness as the effect of an intervention on breathlessness is not important, rather than level of breathlessness and the impact of this on a patient's daily life. As highlighted earlier, refractory breathlessness is indicative of advanced disease. In a systemic review exploring the measurement of breathlessness in advanced disease, Bausewein et al (2007) identified, as well as unidimensional tools such as Borg, a number of multidimensional tools that measured breathlessness. Of these tools they found 11 breathlessness-specific tools and 18 diseasespecific tools. The breathlessness-specific tools $(n=11)$ were found to assess the broader aspects of breathlessness, including activities of daily living, anxiety levels and quality of life, but only 4 were validated in advanced disease and all on cancer patients. Of the 18 multidimensional disease-specific tools, none had been validated in advanced disease. When exploring the tools validated on ILD specifically, only the Dyspnoea-12 tool (Yorke et al, 2011) has been validated on ILD patients. This tool has been developed since the Bausewein et al (2007) review. The assessment tool assesses breathlessness severity, and incorporates both physical and affective aspects of breathlessness in patients with ILD. The tool comprises of 12 descriptor items on the scale and provides an overall score for breathlessness severity that incorporate 7 physical items and 5 affective items. The tool is easy for the patient to complete and quick to use. However, it is important to note the sample size in the validation study was small $(n=101)$, the level of refractory breathlessness was not discussed, and the authors did note the majority of participants had IPF (idiopathic pulmonary fibrosis - a form of ILD) as underlying pathology, questioning the generalisability to patients who have other types of ILD. However, the Dyspnoea-12 tool does appear to have the potential to be an effective assessment tool to use in practice for ILD patients and is worthy of further exploration.

A systematic review of Bausewein et al (2007) also proposed the most effective assessment of breathlessness in advanced disease was a mixed method approach, using both a quantitative tool and a qualitative assessment. This approach does also have limitations, as the reliability of open questioning in this form of assessment cannot be as easily controlled. The clinician could make assumptions and could miss key symptoms if not using a structured framework. In their study, Homsi et al (2006) showed the limitation of using open questioning. They found patients did not volunteer the extent of their symptoms when comparing the answers of patients when using an open questioning approach used by the clinician, to the use a systematic assessment tool, and found a 
considerable number of symptoms were missed. Although this study was undertaken in the cancer field, its relevance is transferable to ILD as it does relate to the symptom burden of advanced disease.

As Johnson et al (2015) identified, the key to effective assessment of breathlessness is the distinction between the perception of the breathlessness intensity and the emotional response of the patient to the breathlessness and the effect this has on quality of life and daily activities. This advocates the use of both quantitative measures and open dialogue.

The emotional burden of breathlessness is well known and breathlessness has been shown to be closely associated to anxiety (Carrieri-Kohlman et al, 2010). However, anxiety is not always included as a component to assess. As there is an integral link between emotion and breathlessness, emotional well-being is important to assess when also reviewing breathlessness.

Therefore, both physical and emotional aspects of breathlessness need to be assessed fully to enable the health care practitioner and the patient to manage breathlessness in partnership.

Rocker (2011) in his editorial concludes that he has 'given up measuring things in favour of just listening to the patients. Just listening commits us to quite a different approach to the assessment of dyspnoea' (Rocker, 2011:219). Given the individual nature of every patient, I would argue this is the only way to truly address their needs.

\section{The management of refractory breathlessness}

Once refractory breathlessness is assessed, how best to manage it is an essential component of care. John's symptoms were managed by adopting a range of pharmacological and non-pharmacological interventions. These included Oramorph', benzodiazepines, antidepressants, oxygen therapy, fan therapy and breathing exercises.

John was treated with pirfenidone and nintedanib; both new antifibrotic drugs in this field. The precise mechanism of pirfenidone is unclear but it is thought it slows down inflammation and the build up of scar tissue in the lungs, thus slowing down the loss of lung function and decreasing disease progression and increasing life expectancy. Nintedanib seems to work similarly by slowing down loss of lung function and reducing the rate of sudden flare-ups. Both these drugs were stopped in recent months as John's lung function continued to decline and he had minimal symptomatic benefit.

Lorazepam three times a day did offer symptomatic relief for John, as did Oramorph, $5 \mathrm{mg}$ every 2-4 hours. Another pharmacological intervention that was given to John was oxygen therapy, with John being administered $8 \mathrm{~L} / \mathrm{min}$ of continuous, humidified, long-term oxygen therapy (LTOT) via a nasal speculum, which is increased to $10 \mathrm{~L} / \mathrm{min}$ on exertion. His resting $\mathrm{SpO}_{2}$ is around $94 \%$ on $8 \mathrm{~L} / \mathrm{min}$. From a non-pharmacological perspective, John found relief from fan therapy when acutely breathless and breathing exercises taught to him by the hospice occupational therapist and physiotherapy teams.

Anxiety and fearful perception of breathlessness has been found to exacerbate the experience and thus perpetuates it (Farquhar et al, 2014). Based on this observation, anxiolytics are used in practice to help manage dyspnoea. However, a meta-analysis by Simon et al (2016) concluded there was insufficient evidence to use benzodiazepines in the management of dyspnoea. They conclude there is a small, non significant trend towards a beneficial effect. Benzodiazepines caused more drowsiness compared to placebo but less compared to morphine. They recommend benzodiazepines to be used when opioids and non-pharmacological measures fail to control breathlessness. They 
conclude that benzodiazepines reduce the sensation of breathlessness through decreasing the anxiety associated with breathlessness.

However, a small scale study undertaken by Allcroft et al (2013) in South Australia with chronic obstructive pulmonary disease (COPD) patients explored the effectiveness of benzodiazepines and sustained release (SR) morphine on breathlessness. The authors found a $15 \%$ reduction using the VAS tool for breathlessness by day 4. The participants in this study were diagnosed with COPD rather than ILD, so generalisability could be questioned, but it does question the evidence base surrounding this intervention.

Abernethy et al's study in 2003 was the first to give definitive benefits for the use of opiates in refractory breathlessness. This was a randomised, double blind, placebo-controlled crossover trial of SR morphine in a group of 48 predominantly COPD patients who were morphine-naïve. They were given $20 \mathrm{mg}$ SR morphine over a 4-day period followed by identically formulated placebo or vice versa. Benefit was measured by improved visual analogue scores, quality of sleep, wellbeing and physical exertion. A total of 38 people completed the study, 5 dropping out due to side effects from the morphine (nausea, vomiting and sedation). Participants scored significantly different dyspnoea scores when treated with morphine compared to placebo.

Currow et al (2014) undertook a narrative review, exploring the use of opioids in refractory breathlessness and concluded low-dose opioids have a key role to play in the safe management of refractory breathlessness. They conclude that patients should be initiated on a dose of $10 \mathrm{mg}$ daily and potentially increasing to $30 \mathrm{mg}$ daily. They highlight there is is a need for further research to address whether upward titration delivers further clinical benefit and whether all underlying aetiologies respond as predictably to opioids. They explore the mechanism of how opioids block the dyspnoea pathway and evaluate the benefits versus harm of using opioids to an individual. In considering measurement of breathlessness they conclude the 'gold standard' for measuring such a multidimensional symptom must be self-assessment by the patient considering impact on intensity, distress and function (Parshall et al, 2012),

John was also prescribed an antidepressant, which he felt lifted his mood and improved his despair. This further illustrates the inter-relationship between the physical and emotion aspects of refractory breathlessness.

The non-pharmacological interventions used included fan therapy. The mechanism by which the fan reduces breathlessness remains unclear. Galbraith et al (2010) suggest the diving response may be a possible explanation: the diving response causes ventilatory depression when the trigeminal area of the face is cooled. Another suggestion is cooling of nasal or oral mucosal receptors and via the decreased central respiratory drive (Marchetti et al, 2005). Although a small study (50 participants), Galbraith et al (2010) showed the benefits of a hand-held fan for relieving dyspnoea in patients with advanced disease. However, the study has a number of limitations, including the use of only a VAS to measure breathlessness. Because it can give immediate self-administered relief for the patient, it would be interesting to explore how the perceived benefits to patients extended to their feelings of mastery of their own condition.

One area of non-pharmacological management that was identified by John and his wife as having huge benefit was the supportive care offered in regular home visits. This gave him an opportunity to share his feelings and be reassured that this is expected in the disease trajectory. To a health care professional (HCP) this can feel like a relatively small contribution, but the reassurance this support provided to John and his wife was essential for them. Increasing frailty in advancing disease has led 
John to question his value, worth and purpose in life. Giving hope and meaning through frequent support visits has encouraged them and reminded them that they are not alone. Haraldsdottir (2011) considered Saunders philosophy of 'being with' a patient, enabling them to examine their thoughts and fears relating to their imminent death. She used ethnomethodology to reveal embedded practice in a hospice with nurses and their patients at end of life, supporting the evidence for sharing the lived experience with patients. The nurses in this study describe their feelings and the challenge of supporting patients at this intimate time of their life.

Higginson et al (2014) carried out a randomised controlled trial (RCT) evaluating an integrated palliative and respiratory care service for patients with advanced disease and refractory breathlessness $(n=105)$. These patients had a range of diseases including cancer, ILD, heart failure and motor neuron disease (all Medical Research Council dyspnoea scale $>2$ ). This was a single blind RCT with patients randomly recruited to either an intervention breathlessness support group and or to receive usual care. The breathlessness support service comprised a multidisciplinary team where assessment and treatment of physical, emotional, psychological and spiritual concerns were through one point of access. Usual care was described as regular NHS care.

Unusually, outcomes were measured primarily by mastery; this was described as the feeling of control over their disease and its effect on quality of life and function. Reduction in dyspnoea was a secondary outcome. It was found that the intervention group scored higher for mastery but there were no significant differences between intervention and control for intensity of average breathlessness. Mastery was on average $16 \%$ higher for those patients receiving the breathlessness support service $(p=0.048)$. The limitations of this study are that it is a single blind trial, the outcome measures were subjective and patients could have been subject to the placebo effect in the breathlessness intervention group. However, patients were unaware that mastery was an endpoint and no mention was made of the pharmacological interventions patients received and how these compared between the control and intervention groups. It is also difficult to quantify normal NHS care as this will vary enormously around the country.

However, this study shows the potential of taking a holistic approach to managing refractory breathlessness. The intervention captured the benefits of a self-management programme and the value of integrated palliative care. However, the study's results have been challenged with Radbruch (2014) questioning if intensity of breathlessness is not improved what was the outcome and how useful is mastery?

Farquhar et al (2014) presented a similar model of a breathlessness intervention service in Cambridge, aiming to break the vicious cycle of anxiety-breathlessness by implementing the breathing-thinking-function intervention. This intervention helps patients set their own goals rather than those identified by an HCP, focusing on achieving improved self-efficacy, motivation and mastery; living better rather than living longer.

\section{Implications for practice}

The medical model values RCTs and quantitative outcome measures as superior research methods, but these will not always capture the essence of some of the benefits from interventions that patients will experience. These may include: peer support, mastery or increased self-worth. These are best described through qualitative evidence. The outcome measures in managing refractory breathlessness are often not sensitive to demonstrating the benefit of an intervention, due to the complex nature of the symptom. Reduction of intensity of breathlessness as the main outcome measure of an intervention is rarely going to be achievable, as these are patients with advancing 
disease with very poor prognoses. However, this does not mean the intervention is not effective as breathlessness is not solely about the physical act of breathing. Taking a psychological approach through a breathlessness intervention service as demonstrated in Cambridge (Farquhar et al, 2014), could equip the individual with self-management tools, and allow them to apply mastery earlier on in the disease journey. If the locus of control sits with the patient from the outset, the patient will be potentially less dependent on HCPs and build up their own physical and emotional resilience. Having hope and meaning at end of life can alter the focus of the patient from 'what's the matter with me' to 'what matters to me'.

Developing a body of evidence in ILD disease-specific assessment tools and intervention strategies is important as evidence shows there are different outcomes for cancer and non-cancer diseases (Homsi et al, 2006). ILD research is a relatively new field of growth and more evidence is needed for supporting these patients throughout their disease and at the end of their lives.

\section{References}

Abernethy AP, Currow DC, Frith P et al. Randomised, double blind, placebo controlled crossover trial of sustained release morphine for the management of refractory dyspnoea. BMJ.

2003;327(7414):523-8

Allcroft P, Margitanovic V, Greene A et al. The role of Benzodiazepines in breathlessness: a single site,open label pilot of sustained release morphine together with clonazepam. J Palliative Med. 2013;16(7)741-4

Bausewein C, Farquhar M, Booth S et al. Measurement of breathlessness in advanced disease: a systematic review. Resp Med. 2007;101(3):399-410

British Lung Foundation. Pulmonary Fibrosis. 2017. https://www.blf.org.uk/support-foryou/pulmonary-fibrosis/what-is-pulmonary-fibrosis (accessed 27 March 2017)

Borg G. Psychophysical bases of perceived exertion. Med Sci Sports Exerc. 1982;14:377-81

British Thoracic Society. Interstitial Lung Disease Registry Programme Annual report 2015/16. 2016. https://www.brit-thoracic.org.uk/document-library/audit-and-quality-improvement/lung-diseaseregistry/bts-ild-registry-annual-report-201516/(accessed 15 August 2017)

Carrieri-Kohlman V, Donesky-Cuenco D, Park SK et al. Additional Evidence for the Affective Dimension of Dyspnoea in Patients with COPD. Res Nurs Health. 2010;33(1):4-19

Currow DC, Quinn S, Greene A et al. The Longitudinal Pattern of Response when Morphine is used to treat Chronic Refractory Disease. J Pall Med. 2013;16(8):881-86

Currow DC, Ekstrom M, Abernethy AP. Opioids for chronic Refractory Breathlessness: right patient, right route? Drugs. 2014;74(1):1-6

Farquhar MC, Prevost AT, McCrone P et al. Is a specialist breathlessness service more effective and cost effective for patient with advanced cancer and their carers than standard care? Findings of a mixed method RCT. BMC Med. 2014;12(194):1-13

Galbraith S, Fagan P, Perkins P et al. Does the use of a handheld fan improve chronic dyspnoea? A randomised, controlled, cross over trial. J Pain Symptom Manage. 2010;39(5):831-38 
Gracely RH, Undem BJ, Banzett RB. Cough, pain and dyspnoea: similarities and differences. Pulm Pharmacol Ther. 2007;20:433-7

Haraldsdottir E. The constraints of the ordinary :'being with ' in the context of end of life nursing care. Int J Pall Nurs. 2011;17:245-50

Herigstad M, Hayen A, Wiech K, Pattinson KT. Dyspnoea and the brain. Respir Med. 2011;105(6):809-17

Higginson IJ, Bausewein C, Reilly CC et al. An integrated palliative and respiratory care service for patients with advanced disease and refractory breathlessness: a randomised controlled trial. Lancet Respir Med. 2014;2(12):979-87

Homsi J, Walsh D, Rivera $\mathrm{N}$ et al. Symptom evaluation in palliative medicine: patient report vs systematic assessment. Support Care Cancer. 2006;14(5): 444-53

Janssens T, Verleden G, De Peuter S et al. Inaccurate perception of asthma symptoms: a cognitiveaffective framework and implications for asthma treatment. Clin Pyschol Rev. 2009;29(4):317-27

Johnson MJ, Currow DC, Booth S. Prevalence and assessment of breathlessness in the clinical setting. Expert Rev Respir Med. 2014;8(2)151-61

Johnson MJ, Kanaan M, Richardson $\mathrm{G}$ et al. A randomised controlled trial of three or one breathing technique training sessions for breathlessness in people with malignant lung disease. BMC Med. 2015;13:213

Marchetti $\mathrm{N}$ et al (2005) Effect of air directed to the face on diaphragm electromyographic activity (EMGDia) during hypercapnic challenge (abstract) Proceedings of the 100th Anniversary International Conference American Thoracic Society May 20-25 San Diego CAA789

McCord M, Chronin-Stubbs D. Operationalising dyspnoea: focus on measurement. Heart Lung. 1992;21(2):167-79

National Institute for Health and Care Excellence. TA282: Pirfenidone for treating idiopathic pulmonary fibrosis. 2013. http://www.nice.org.uk/guidance/TA282 (accessed on 27 March 2017)

National Institute for Health and Care Excellence .TA379: Nintedanib for treating idiopathic pulmonary fibrosis. 2016. http://www. nice.org.uk/guidance/TA379 (accessed on 27 March 2017)

Parshall MB, Schwartzstein RM, Adams L. An Official American Thoracic Society Statement: Update on the Mechanisms, Assessment, and Management of Dyspnoea. Am J Respir Crit Care Med.

2012;185(4):435-52

Radbruch L. Mastering breathlessnes in patients with advanced respiratory disease. Lancet.

2014;2:944-5

Rocker G. Dyspneoa: recent insights and innovations. J Prog Pall Care. 2014;19(5):219-22

Roper N, Logan W, Tierney A. Activities of Living: a model for nursing. Nurs Times. 1983;79(9):24-7

Simon ST, Higginson IJ, Booth S et al. Benzodiazepines for the relief of breathlessness in advanced malignant and non-malignant diseases in adults. Cochrane Database Syst Rev. 2016;10:CD007354

Wilson RC, Jones PW. A comparison of the visual analogue scale and modified Borg scale for the measurement of dyspnoea during exercise. Clinic Scient. 1989;76(3):277-82 
Yorke J, Swigris J, Russell AM. Dyspnoea-12 is a valid and reliable measure of breathlessness in patients with Interstitial Lung Disease. Chest. 2011;139(1):159-64 